\title{
Antipsychotics Associated with the Development of Type 2 Diabetes in Antipsychotic-Naïve Schizophrenia Patients
}

\author{
Jimmi Nielsen*,', S $\varnothing$ ren Skadhede' and Christoph U Correll ${ }^{2,3}$ \\ 'Unit for Psychiatric Research, Aalborg Psychiatric Hospital, Aarhus University Hospital, Mølleparkvej 10, Aalborg, Denmark; ${ }^{2}$ The Zucker Hillside \\ Hospital, Psychiatry Research, North Shore-Long Island Jewish Health System, Glen Oaks, NY, USA; ${ }^{3}$ Albert Einstein College of Medicine, Bronx, \\ NY, USA
}

\begin{abstract}
Diabetes mellitus occurs in schizophrenia patients at higher rates than in the general population. Reasons for this elevated risk are poorly understood and have not been examined prospectively in antipsychotic-naïve, first-episode patients. This study aims to determine which antipsychotics are associated with diabetes development in antipsychotic-naïve schizophrenia patients. All antipsychotic-naïve patients diagnosed with schizophrenia in Denmark between 0 I January 1997 and 3 I December 2004, followed until 3I December 2007, allowing for $\geqslant 3$ years follow-up, unless death or diabetes onset occurred. Risk factors for the time to diabetes onset were assessed, including antipsychotics taken for at least 180 defined daily doses in the first year after first antipsychotic prescription ('initial treatment'). Risk factors for diabetes incidence were assessed, including antipsychotic use within 3 months before diabetes onset or study end ('current treatment'). Of 7139 patients, followed for 6.6 years (47 297 patient years), 307 developed diabetes (annual incidence rate: $0.65 \%$ ). Time to diabetes onset was significantly shorter in patients with higher age (hazard ratio (HR): I.03, confidence interval (Cl): I.02-।.03) and those with 'initial' treatment of olanzapine (HR: I.4I, Cl: I.09-I.83), mid-potency first-generation antipsychotics (FGAs) (HR: I.60, Cl: I.07-2.39), antihypertensive (HR: 1.87, Cl: I.13-3.09), or lipid-lowering drugs (HR: 4.67, Cl: 2. 19-10.00). Significant factors associated with diabetes within 3 month of its development included treatment with low-potency FGAs (odds ratio (OR): I.52, Cl: I.I4-2.02), olanzapine (OR: I.44, Cl: 1.98-1.91), and clozapine (OR: 1.67, Cl: I.14-2.46), whereas aripiprazole was associated with lower diabetes risk (OR: 0.5I, Cl: 0.33-0.80). In addition to general diabetes risk factors, such as age, hypertension, and dyslipidemia, diabetes is promoted in schizophrenia patients by initial and current treatment with olanzapine and mid-potency FGAs, as well as by current treatment with or low-potency first-generation antipsychotics and clozapine, whereas current aripiprazole treatment reduced diabetes risk. Patients discontinuing olanzapine or mid-potency FGA had no increased risk of diabetes compared with patient not treated with the drugs at anytime.

Neuropsychopharmacology (2010) 35, 1997-2004; doi:I0.1038/npp.2010.78; published online 2 June 2010
\end{abstract}

Keywords: schizophrenia; first-episode; diabetes; olanzapine; clozapine; first-generation antipsychotics

\section{INTRODUCTION}

Patients with schizophrenia have a reduced lifespan of $>20$ years, with cardiovascular disorders being the main cause of death (Colton and Manderscheid, 2006; Tiihonen et al, 2009). Reasons for the premature cardiovascular death are related to higher rates of cardiovascular risk factors in patients with severe mental disorders, including obesity, arterial hypertension, the metabolic syndrome, and type 2 diabetes (Newcomer, 2005). Reasons for the increased prevalence of cardiovascular risk factors are complex and

*Correspondence: Dr J Nielsen, Unit for Psychiatric Research, Aalborg Psychiatric Hospital, Aarhus University Hospital, Mølleparkvej I0, PO Box 210, Aalborg DK-9100, Denmark, Tel: + 452577 9926,

E-mail: jin@rn.dk

Received 26 March 20I0; revised 20 April 2010; accepted 27 April 2010 include sedentary lifestyle, (Koopman et al, 2005), treatments that increase body weight and worsen metabolic parameters (Newcomer, 2005) and, possibly, illness-related or genetic factors (Fleischhacker et al, 2008).

Diabetes mellitus is a particularly strong risk factor for cardiovascular morbidity, being a risk equivalent of myocardial infarction (Haffner et al, 1998). Despite their widespread use, second-generation antipsychotics (SGAs), especially olanzapine and clozapine, have been associated with metabolic syndrome and the development of type 2 diabetes (Cohen and Correll, 2009; Meyer and Stahl, 2009; Yood et al, 2009). However, the risk of metabolic complications differs within the class of first-generation antipsychotics (FGAs) and SGAs, with high-potency FGAs and more recent SGAs, such as aripiprazole and ziprasidone, having smaller metabolic burden compared with the rest of the antipsychotic drugs (Meyer et al, 2008). 
Antipsychotic-induced weight gain is considered as an indirect pathway to the development of diabetes through decreasing peripheral insulin resistance because of decreased insulin sensitivity. Of note, in addition to indirect pathways to diabetes development, at least, studies with olanzapine and clozapine have suggested a possible, direct adverse effect on glucose homeostasis (Henderson et al, 2006; Houseknecht et al, 2007; Kinon et al, 2008; Newcomer et al, 2002). Moreover, in a recent study of antipsychoticnaïve youth treated with olanzapine, the adverse metabolic effects on glucose and lipid metabolism seemed to be dose related, whereas increases in body composition parameters were not dose related (Correll et al, 2009). This opens the possibility that a direct diabetogenic effect of olanzapine may accelerate the development of diabetes in susceptible individuals even more when occurring early in the treatment course, leading to a stronger signal compared with other antipsychotics than when given later in the illness course, when weight gain associated with other medications is added to the pathophysiology of diabetes development.

As diabetes development in the severely mentally ill is an increasing issue of high public health importance, we aimed to identify antipsychotics associated with increased risk for the development of diabetes in patients with first-episode schizophrenia. This information is relevant to develop strategies that decrease the risk of metabolic complications in severely mentally ill patients. To date, diabetes development has not been studied as an outcome in first-episode cohorts because of the small numbers and relatively brief follow-up periods. Therefore, we used a cohort study design, using a large and representative data set. We focused on patients with first-episode schizophrenia to decrease the confounding effect of unknown prior treatments and to examine whether the early use of antipsychotics with more pronounced cardiometabolic effects is associated with an acceleration of diabetes development that usually takes many years to develop. We hypothesized that use of olanzapine during the first year after initiation of antipsychotics and current use of olanzapine or clozapine would be associated with the development of diabetes.

\section{MATERIALS AND METHODS}

\section{Sample}

This cohort study was approved by the Danish Data Protection Agency, National Board of Health and Statistics Denmark. Subjects for the analyses were identified by having an onset of an ICD-10 F20 schizophrenia diagnosis in the Danish Central Psychiatric Research Registry (Munk-Jorgensen and Mortensen, 1997) in the period from 01 January 1997 until 31 December 2007. The register has a high validity and has contributed significantly to epidemiological research (Barr et al, 1990; Bock et al, 2009; Laursen et al, 2005; Pedersen and Mortensen, 2001; Qin and Nordentoft, 2005).

Prescription data for the included subjects were obtained from the national prescription database from 01 January 1996 through 31 December 2007. Prescription data were assessed at least 1 year before onset of schizophrenia to exclude cases with a history of diabetes before the first prescription of antipsychotics. Index date was the date of first antipsychotic prescription ever, even if this predated the formal diagnosis of schizophrenia. To ensure a minimum of 3 years of follow-up, subjects having a first antipsychotic prescription after 31 December 2004 were excluded. Only subjects receiving at least one prescription of an antipsychotic were included in the analysis. The prescription database contains information about number of sold daily defined doses (DDDs), which are assigned and reviewed by researchers of the World Health Organization Collaborating Centre of Drug Statistics Methodology (WHO, 2009). Subjects with a history of any psychotic disease before 01 January 1997 (ie ICD-10: F2 and ICD-8: 295-299) were also excluded. Follow-up time was defined as time from first prescription of antipsychotics until onset of diabetes, death, or end of register 31 December 2007.

Diabetes onset was defined as receiving a diagnosis of type 2 diabetes (ICD-10: E11) from a somatic hospital or outpatient clinic, or filling at least one prescription for an oral antidiabetic (ATC: A10B), whichever came first. Patients with diabetes onset before first antipsychotic prescription $(N=91)$ were excluded from further analyses. Prescription of insulin was not used in the definition of diabetes onset because of the risk of including subjects with a type 1 diabetes. Furthermore, insulin is rarely used as the only antidiabetic agent for type 2 diabetes. In this dataset, only five cases received insulin alone after the onset of antipsychotic prescription, and these were also excluded.

\section{Statistical Analyses}

Statistical analyses were performed with STATA 10 at Statistics Denmark server through remote access. As cardiovascular risk factors, for example age, sex, obesity, hypertension, and dyslipidemia, contribute to the development of type 2 diabetes, the following variables were used in the multivariate statistical models in addition to antipsychotic medications: age, sex, receiving lipid-lowering medications, or antihypertensive medications. Furthermore, two variables were created as a proxy measure of severity of illness: percentage of follow-up time as an inpatient and average antipsychotic DDD during follow-up. Days with inpatient status were excluded from the calculation of average DDD because medications dispensed during hospitalization are not included in the national prescription database.

Antipsychotics were divided into FGA and SGA, and FGA were subdivided in low, mid- and high-potency FGA. The low-potency FGA group consisted of chlorpromazine, levomepromazine, chlorprothixen, melperone, and pipamperone. The mid-potency FGA group consisted of zuclopenthixol, periciazine, prochlorperazin, and perphenazine. The high-potency FGA group consisted of haloperidol, pimozide, flupenthixol, and fluphenazine. The SGA group consisted of olanzapine, quetiapine, sertindole, amisulpride, risperidone, ziprasidone, sulpride, and clozapine.

'Initial' antipsychotic treatment was defined as receiving at least 180 DDD of the same antipsychotic drug within the first 365 days after first antipsychotic prescription, which could predate the diagnosis of schizophrenia. Bed days were excluded in this definition, as inpatient medications were not available. 'Current' antipsychotic treatment was defined 
as filling at least one prescription of an antipsychotic drug within the last 3 months period before diabetes onset, death, or end of registry. If patients were on more than one antipsychotic, diabetes risk counted to each antipsychotic.

In addition, continuation of the type of antipsychotics 3 months before and after onset of diabetes was determined to assess whether patients were continued on diabetogenic antipsychotic drugs. Aripiprazole and ziprasidone are considered as low metabolic risk antipsychotics, which make them suitable for first-line treatment and for metabolically vulnerable patients. Earlier treatment with low metabolic risk antipsychotic was assessed to determine whether treatment with these drugs had been attempted before the use of higher metabolic risk antipsychotics.

Age of diabetes onset and age at last observation were transformed by square root and transformed back to obtain the mean and $95 \%$ confidence intervals (CIs). Age of schizophrenia and age of first antipsychotic prescription were transformed by the natural logarithm. Percentages of psychiatric inpatient time were not normally distributed without the possibility for transformation. Consequently, the non-parametric Wilcoxon's rank sum test was used to compare rates between patients with and without diabetes. Binary variables were tested with a $\chi^{2}$ test.

The effect of initial treatment variables on the time to onset of diabetes was tested using the Cox regression model that was adjusted for sex, and age of first antipsychotic prescription. The effect of current treatment variables was tested in a multiple regression model. Variables entered into the Cox model and the multiple regression model were those significantly associated with diabetes determined by stepwise forward logistic regression, including variables with $P<0.1$, followed by a stepwise backward logistic regression excluding variables with $P>0.05$. For the multiple regression model of current antipsychotic use, both Crude and odds ratios (ORs) adjusted for observation time were calculated. This was performed because of the continuous introduction of atypical antipsychotics during the study period. Antipsychotics with initial use associated with an increased risk of diabetes were divided in subjects who continued the treatment for $>1$ year and those who discontinued treatment. The discontinuers were compared with subjects never treated with the drug. In the multivariate models, the following continuous variables were treated as discrete variables: age, percentage of psychiatric inpatient time, and DDD. This was performed to be able to calculate (ORs) and hazard ratios (HRs), which indicate and increase in age from year to year, in percent of time hospitalized by $1 \%$ (eg $2-3 \%$ and so on), and in DDD by 1 DDD (ie 1 vs 2 DDD and so on).

\section{RESULTS}

Altogether, 7139 first-episode schizophrenia subjects (mean age at first antipsychotic prescription: 29.7 years, 59.7\% male) without diabetes before first antipsychotic prescription were included in this study, with a total observation time of 47297 patient years and a mean observation time of $6.6(\mathrm{SD}=2.4)$ years. Table 1 displays patient demographics as well as characteristics of the initial treatment and the treatment during the last 3 months. In total, 307 patients developed diabetes during the follow-up, translating into an annual diabetes incidence rate of $0.65 \%$ per patient year. The mean age of onset of type 2 diabetes was 41.7 years (40.4-43.1), and the median onset after first antipsychotic prescription was 3.9 years (25 and 75 percentiles: 2.3 and 6.0 years).

\section{Effect of Variables Present during the First Year of Antipsychotic Treatment}

In the Cox regression model, time to diabetes onset was significantly shorter in patients with higher age (HR: 1.03, CI: 1.02-1.03) and those with 'initial' treatment of olanzapine (HR: 1.41, CI: 1.09-1.83), mid-potency FGAs (HR: 1.60, CI: 1.07-2.39), antihypertensive (HR: 1.87, CI: 1.13-3.09), or lipid-lowering drugs (HR: 4.67, CI: 2.19-10.00) (Table 2). Low-potency FGAs also had a trend toward an increased risk of diabetes, but remained insignificant (OR: 1.63, CI: 0.93-2.84, $p>0.086$ ). Discontinuers of olanzapine had no increased risk compared with patients not receiving olanzapine at anytime within the study period. Similar result was found for mid-potency FGA.

\section{Effect of Variables Present during the Entire Follow-Up and of Treatment Variables Present during the Last 3 Months Before Diabetes Onset or at Study Completion}

In the adjusted multivariate model, significant factors associated with diabetes incidence within 3 month of its development included 'current' treatment with low-potency FGAs (OR: 1.56, CI: 1.17-2.07), olanzapine (OR: 1.96, CI: 1.48-2.58), and clozapine (OR: 2.48, CI: 1.68-3.66), whereas aripiprazole (OR: $0.51, \mathrm{CI}: 0.36-0.86$ ), not receiving antipsychotics (OR: 0.57 CI: $0.41-0.79$ ), and male sex (OR: 0.71 , CI: $0.55-0.90)$ were associated with lower diabetes incidence (Table 3).

Neither sole treatment with either SGAs or FGAs during the entire follow-up period was associated with an increased risk of diabetes. Higher dosages of antipsychotics during follow-up were associated with an increased risk for diabetes (OR: 1.10, CI: $1.02-1.19, p<0.01$ ) only in a univariate logistic regression model.

\section{Antipsychotic Treatment at the Time of Diabetes Onset and 3 Months Later}

Patients developing diabetes were to a large extent on high metabolic risk antipsychotics, such as olanzapine (27.7\%), low-potency FGAs (25.1\%), and clozapine (11.7\%) during diabetes onset. Three months after diabetes onset, $84 \%$ of patients treated with olanzapine, $94 \%$ with clozapine, $91 \%$ with quetiapine, $86 \%$ with risperidone, and $86 \%$ treated with low- or mid-potency FGAs remained on these medications. During the same time frame, only eight (4\%) and five $(2 \%)$ patients had initiated treatment with one of the two low metabolic risk antipsychotics, aripiprazole, and ziprasidone, respectively. Of patients with diabetes, only 80 (26.1\%) had earlier been treated with one of the low-risk metabolic drugs, that is aripiprazole: $41(13.4 \%)$ and ziprasidone: 53 (17.6\%). 
Table I Demographic, Illness, and Treatment Characteristics

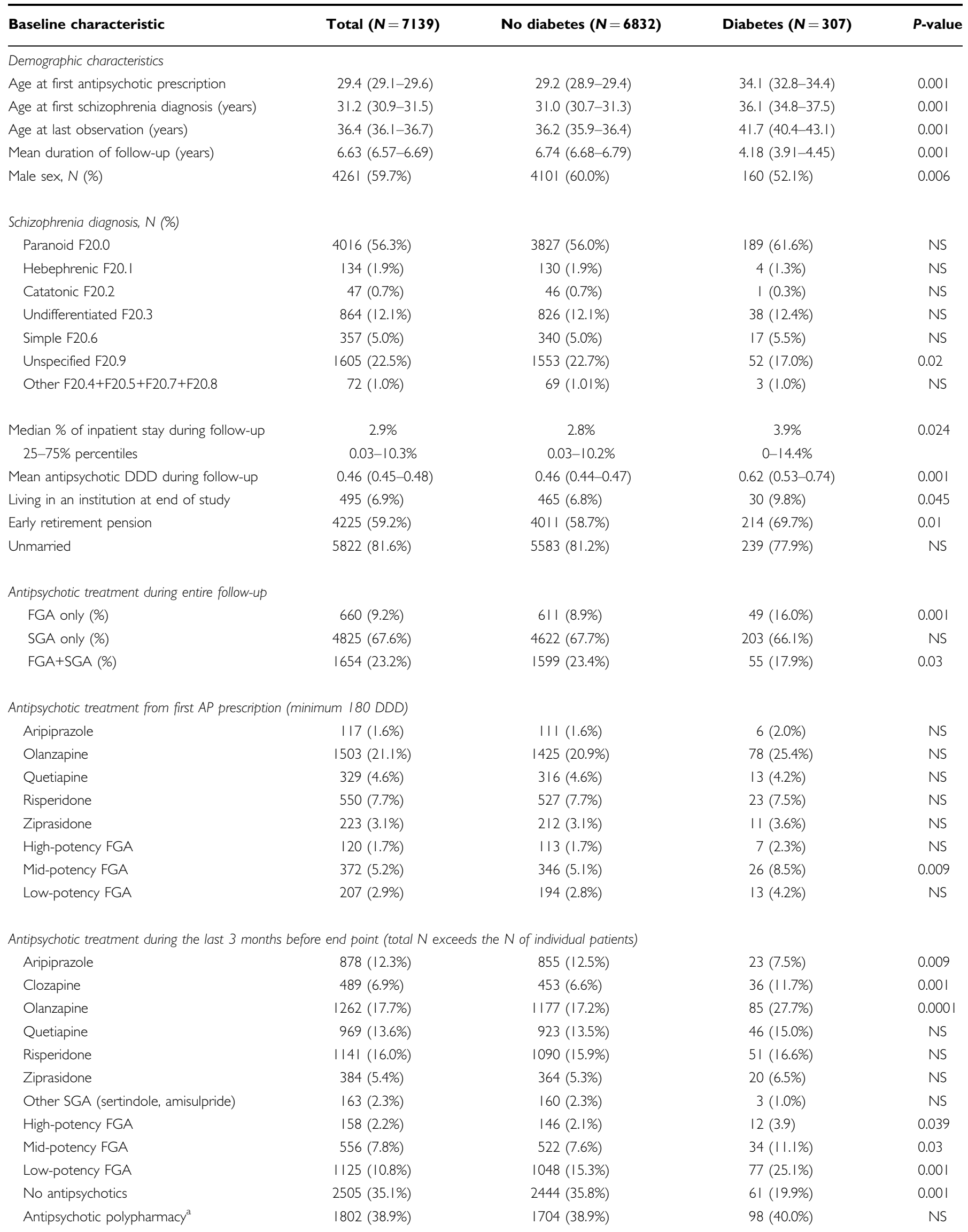


Table I Continued

\begin{tabular}{|c|c|c|c|c|}
\hline Baseline characteristic & Total $(N=7139)$ & No diabetes $(N=6832)$ & Diabetes $(N=307)$ & P-value \\
\hline \multicolumn{5}{|c|}{ Non-antipsychotic treatment the first year after first AP prescription } \\
\hline Anxiolytics/hypnotics, N (\%) & $3906(54.7 \%)$ & $3696(54.1 \%)$ & $210(68.4 \%)$ & 0.001 \\
\hline Antidepressants, $N(\%)$ & $4298(60.2 \%)$ & $4083(59.8 \%)$ & $215(70.0 \%)$ & 0.001 \\
\hline \multicolumn{5}{|l|}{ Mood stabilizers } \\
\hline Valproic acid/divalproex, N (\%) & $136(1.9 \%)$ & $130(1.9 \%)$ & $6(1.9 \%)$ & NS \\
\hline Lipid-lowering drugs, N (\%) & $27(0.4 \%)$ & $20(0.3 \%)$ & $7(2.3 \%)$ & 0.001 \\
\hline Antihypertensive drugs, $N(\%)$ & $150(2.1 \%)$ & $132(1.9 \%)$ & I 8 (5.9\%) & 0.001 \\
\hline \multicolumn{5}{|c|}{ Non-antipsychotic treatment 3 months before end point } \\
\hline Anxiolytics/hypnotics, N (\%) & $2287(32.0 \%)$ & $2159(31.6 \%)$ & $128(41.7 \%)$ & 0.001 \\
\hline Lipid-lowering drugs, $N(\%)$ & $177(2.5 \%)$ & $145(2.1 \%)$ & $32(10.4 \%)$ & 0.001 \\
\hline Antihypertensive drugs, N (\%) & $121(1.7 \%)$ & $110(1.6 \%)$ & II (3.6\%) & 0.009 \\
\hline \multicolumn{5}{|c|}{ Low metabolic risk drugs prescribed at anytime during follow-up } \\
\hline Aripiprazole & I 803 (25.3\%) & $1762(25.8 \%)$ & $4 \mid(13.4 \%)$ & 0.001 \\
\hline Ziprasidone & $1528(21.4 \%)$ & $1475(21.6 \%)$ & $53(17.3 \%)$ & NS \\
\hline Aripiprazole or ziprasidone & $2695(37.8 \%)$ & $2615(38.3 \%)$ & $80(26.1 \%)$ & 0.001 \\
\hline High-potency FGA & $1 \mid 12(15.6 \%)$ & $1060(\mid 5.5 \%)$ & $52(16.9 \%)$ & NS \\
\hline
\end{tabular}

apercentage is only of those receiving antipsychotics within the last 3 months before end point.

Table 2 Cox Regression Showing Variables during Initial Treatment Affecting Time until Onset of Diabetes

\begin{tabular}{lrrrrr}
\hline Variable & HR & $\mathbf{z}$ & $\boldsymbol{P}$ & \multicolumn{2}{c}{$\mathbf{9 5 \%} \mathbf{~ C l}$} \\
\hline Male gender & 0.80 & -1.95 & 0.051 & 0.63 & 1.00 \\
Age of first prescription of antipsychotics & & & \\
Olanzapine & 1.02 & 5.96 & 0.001 & 1.02 & 1.03 \\
Mid-potency FGA & 1.41 & 2.62 & 0.009 & 1.09 & 1.83 \\
Receiving antihypertensive medication & 1.60 & 2.28 & 0.023 & 1.07 & 2.39 \\
Receiving lipid-lowering medication & 1.87 & 2.45 & 0.014 & 1.13 & 3.09 \\
& 4.67 & 3.97 & 0.001 & 2.19 & 10.00 \\
\hline
\end{tabular}

AP: antipsychotic; FGA: first-generation antipsychotic.

aPercentage of time being hospitalized is used as a continuous variable, that is odds ratio (OR) indicates increase from, for example, 2 to $3 \%$ and so on.

\section{DISCUSSION}

The results from this largest cohort study of antipsychoticnaïve schizophrenia patients indicate that in addition to general diabetes risk factors, such as higher age, hypertension, and dyslipidemia (indicated by treatment received for these conditions) (Narayan et al, 2003; Lorenzo et al, 2003), diabetes is promoted by initial treatment with olanzapine and mid-potency FGAs, as well as by current treatment with low-potency FGAs, olanzapine, and clozapine, whereas current aripiprazole treatment and male sex were associated with a reduced diabetes risk.

The finding that the current use of the SGAs, olanzapine, and clozapine increases the risk of diabetes is consistent with earlier findings (Gianfrancesco et al, 2002; Ramaswamy et al, 2006; Smith et al, 2008; Stahl et al, 2009; Yood et al, 2009). However, to our knowledge, this is the first study showing that the metabolic effects of olanzapine early or during treatment can alter the trajectory to diabetes. This could be explained by the fact that the weight that was gained initially is maintained throughout the course of the illness, adversely affecting insulin resistance. Alternatively, it is also possible that direct effects on the pancreatic $\beta$ cell predispose vulnerable individuals to an earlier diabetes onset. These findings call into question the use of olanzapine as a first-line treatment for firstepisode schizophrenia (Moore et al, 2007) and underscore that the anticipated benefits of using olanzapine early on in the treatment algorithm should clearly outweigh the increased risk for diabetes; otherwise lower metabolic risk antipsychotics should be used.

In contrast, the situation for clozapine use differs because it is indicated for treatment-resistant schizophrenia and reserved for the most severely ill, refractory patients (Nielsen et al, 2009; Taylor et al, 2003), which can affect the risk for diabetes through perhaps even poorer healthy lifestyle behaviors than is common in schizophrenia patients in general (Sharpe et al, 2006). Furthermore, the 
Table 3 Factors Associated with Altered Risk for Type 2 Diabetes during the Last 3 Months of Follow-Up

\begin{tabular}{|c|c|c|c|c|c|c|c|c|c|c|}
\hline \multirow{2}{*}{$\begin{array}{l}\text { Variable } \\
\text { Aripiprazole }\end{array}$} & \multicolumn{5}{|c|}{ Crude } & \multicolumn{5}{|c|}{ Adjusted for observation time } \\
\hline & $\begin{array}{c}\text { OR } \\
0.51\end{array}$ & $\begin{array}{c}\mathbf{z} \\
-2.95\end{array}$ & $\begin{array}{c}\boldsymbol{p} \\
0.003\end{array}$ & \multicolumn{2}{|c|}{$95 \% \mathrm{Cl}$} & $\frac{\text { OR }}{0.53}$ & $\begin{array}{c}\mathbf{z} \\
-2.8\end{array}$ & $\begin{array}{c}\mathbf{p} \\
0.005\end{array}$ & \multicolumn{2}{|c|}{$95 \% \mathrm{Cl}$} \\
\hline Receiving no antipsychotics & 0.57 & -3.36 & 0.001 & 0.41 & 0.79 & 0.60 & -2.97 & 0.003 & 0.43 & 0.84 \\
\hline Percentage of time being hospitalized ${ }^{a}$ & 1.02 & 3.89 & 0.001 & 1.01 & 1.02 & NS & & & & \\
\hline Low-potency FGA & 1.52 & 2.84 & 0.005 & 1.14 & 2.02 & 1.45 & 2.44 & 0.015 & 1.08 & 1.96 \\
\hline Clozapine & 1.67 & 2.61 & 0.009 & 1.14 & 2.46 & 2.31 & 4.12 & 0.001 & 1.55 & 3.44 \\
\hline
\end{tabular}

AP: antipsychotic; FGA: first-generation antipsychotic.

aPercentage of time being hospitalized is used as a continuous variable, that is odds ratio (OR) indicates increase from, for example, 2 to $3 \%$ and so on.

superior efficacy of clozapine in treatment refractory patients (Kane et al, 1988) indicates that the increased diabetes risk has to be balanced with the chance for enhanced efficacy.

Initial treatment with mid-potency FGAs and current treatment with low-potency FGAs was also associated with an increased risk for diabetes. This finding is somewhat at odds with a recent meta-analysis (Smith et al, 2008) that found a significantly increased risk for diabetes with SGAs compared with FGAs (OR: 1.13, CI: 1.15-1.51). However, in this meta-analysis, insufficient data were available for ziprasidone and aripiprazole, the latter of which was associated with a lower risk for diabetes during current use in our study. This result, together with our finding of the independently increased risk for diabetes with low- and mid-potency FGAs highlights the fact that diabetes development is not solely an issue with FGAs, and, more importantly, that SGAs and FGAs are heterogeneous classes that should not be lumped together. Initial treatment with low-potency FGA was not associated with an increased risk of diabetes, which might be due to low numbers. Rather, medications should be considered separately, or grouped by individual cardiometabolic risk, which cuts across SGAs and FGAs (Citrome et al, 2007; Hagg et al, 1998).

In this study, the mean age of diagnosis of schizophrenia is somewhat higher than found in other studies in which the age is generally around the mid-twenties (Bertelsen et al, 2009; Kahn et al, 2008; Robinson et al, 1999). However, these studies usually have an upper age limit of around 40 years and/or also focus on first-episode psychosis instead of first-episode schizophrenia, explaining the lower mean age compared with our study in which no upper age limit was set. In our study, the mean age of diabetes onset was around 41 years. This is lower than in the general population and similar to the results from earlier studies with antipsychotics (Cohen et al, 2006). In the first half of the last century, female sex was considered as risk factor for type 2 diabetes, but this difference had vanished by the end of the last century (Gale and Gillespie, 2001). However, in a review of pharmacoepidemiologic studies of antipsychotic use and diabetes, an association with female sex was also found similarly in our study (Ramaswamy et al, 2006). Future studies should investigate sex differences regarding the antipsychotic effects and lifestyle influences that could affect diabetes risk in antipsychotic-treated individuals.

Of note, a high rate of the patients developing diabetes during treatment with high-risk metabolic drugs, olanzapine and clozapine, were maintained on this treatment 3 months after diabetes onset, suggesting that either switching to a lower-risk metabolic drug was not clinically feasible, or that psychiatrists were not considering this option. It is further possible that clinicians did not believe that removing the high metabolic risk antipsychotic would reverse the diabetes, even though this has been described in case series before (De Hert et al, 2006). Similarly, fewer patients with diabetes had been treated with a low metabolic risk antipsychotic in the past compared with patients who did not develop diabetes, implying that some cases of diabetes might have been prevented or postponed by using lower cardiometabolic risk antipsychotics, especially in the early illness course. This is supported by our finding that olanzapine discontinuers had no increased risk of diabetes.

The findings of this study have to be interpreted within its limitations. These include the observational study design, lack of randomized treatment assignment, lack of a control group, and potential influence of unmeasured and unknown confounding factors that cannot be controlled for, such as family history of diabetes, diet and exercise behaviors, body composition, medication adherence, severity of psychiatric illness, and changes in prescribing patterns. Moreover, findings are associations and causal inferences cannot be made. In addition, we only studied in detail the influence of initial and current treatment, and treatment periods in between might have also influenced the outcome. Furthermore, the metabolic screening procedure might have differed between the antipsychotic drugs, for example clozapine patients might have had more frequent blood draws because of the mandatory hematological monitoring. In contrast, patients treated with low metabolic risk drugs, for example aripiprazole, might have had less monitoring because of psychiatrists' perception that these drugs are not causing diabetes and this could have delayed the diabetes diagnosis. Unfortunately, we did not have access to measure the screening procedure. Furthermore, it is not fully possible to control for confounding by indication, that is choosing a low-risk metabolic drug for a metabolically 
vulnerable patient. However, despite this potential bias, current use of aripiprazole was still associated with a reduced diabetes risk. Despite these limitations, the size of this sample, the relatively long-term follow-up duration, focus on first-episode schizophrenia patients, and the follow-up from the beginning of antipsychotic prescribing in earlier antipsychotic-naïve subjects are clear strengths of this study.

On the basis of the results from this and earlier studies, clinicians should focus on preventing initial weight gain because subsequent long-term weight loss is difficult to achieve, either through behavioral or pharmacologic (Marder et al, 2004) interventions. Moreover, all patients receiving antipsychotics should undergo routine monitoring of weight and metabolic parameters (ADA, 2004), and, preferentially, medications with low metabolic liabilities should be used (Meyer and Stahl, 2009). Given the recent data that monitoring has not increased after the warnings about metabolic risks of antipsychotics (Morrato et al, 2010), educational actions and quality control and improvement initiatives should be taken and studied to improve cardiometabolic outcomes in the vulnerable schizophrenia patients. Finally, from a metabolic syndrome point of view, patient should initially receive treatment with antipsychotics possessing a low risk for weight gain to minimize the burden of metabolic side effects.

\section{ACKNOWLEDGEMENTS}

Jimmi Nielsen had full access to all of the data in the study and takes responsibility for the integrity of the data and the accuracy of the data analysis. This study was funded by Aalborg Psychiatric Hospital, which had no influence on data analysis and preparation of the manuscript.

\section{DISCLOSURES/CONFLICT OF INTEREST}

J Nielsen has received research grants from $H$ Lundbeck for clinical trials and received speaking fees from Bristol-Myers Squibb, Astra Zeneca, Janssen Pharmaceutica, and Eli-Lilly. Søren Skadhede has no financial disclosures. CU Correll has been a consultant to or has received honoraria from Actelion, AstraZeneca, Bristol-Myers Squibb, Cephalon, Eli Lilly, GSK, Hoffmann-La Roche, Lundbeck; MedAvante; Medicure; Janssen/J\&J, Schering-Plough; Otsuka, Pfizer, Supernus; Takeda; and Vanda.

\section{REFERENCES}

ADA (2004). Consensus development conference on antipsychotic drugs and obesity and diabetes. Diabetes Care 27: 596-601.

Barr CE, Mednick SA, Munk-Jorgensen P (1990). Exposure to influenza epidemics during gestation and adult schizophrenia. A 40-year study. Arch Gen Psychiatry 47: 869-874.

Bertelsen M, Jeppesen P, Petersen L, Thorup A, Ohlenschlaeger J, Le Quach P et al (2009). Course of illness in a sample of 265 patients with first-episode psychosis - five-year follow-up of the Danish OPUS trial. Schizophr Res 107: 173-178.

Bock C, Bukh JD, Vinberg M, Gether U, Kessing LV (2009). Validity of the diagnosis of a single depressive episode in a case register. Clin Pract Epidemol Ment Health 5: 4.
Citrome LL, Holt RI, Zachry WM, Clewell JD, Orth PA, Karagianis JL et al (2007). Risk of treatment-emergent diabetes mellitus in patients receiving antipsychotics. Ann Pharmacother 41: 1593-1603.

Cohen D, Correll CU (2009). Second-generation antipsychoticassociated diabetes mellitus and diabetic ketoacidosis: mechanisms, predictors, and screening need. J Clin Psychiatry 70: 765-766.

Cohen D, Stolk RP, Grobbee DE, Gispen-de Wied CC (2006). Hyperglycemia and diabetes in patients with schizophrenia or schizoaffective disorders. Diabetes Care 29: 786-791.

Colton CW, Manderscheid RW (2006). Congruencies in increased mortality rates, years of potential life lost, and causes of death among public mental health clients in eight states. Prev Chronic Dis 3: A42.

Correll CU, Manu P, Olshanskiy V, Napolitano B, Kane JM, Malhotra AK (2009). Cardiometabolic risk of second-generation antipsychotic medications during first-time use in children and adolescents. JAMA 302: 1765-1773.

De Hert M, Hanssens L, van Winkel R, Wampers M, Van Eyck D, Scheen A et al (2006). Reversibility of antipsychotic treatmentrelated diabetes in patients with schizophrenia: a case series of switching to aripiprazole. Diabetes Care 29: 2329-2330.

Fleischhacker WW, Cetkovich-Bakmas M, De Hert M, Hennekens $\mathrm{CH}$, Lambert $\mathrm{M}$, Leucht $\mathrm{S}$ et al (2008). Comorbid somatic illnesses in patients with severe mental disorders: clinical, policy, and research challenges. $J$ Clin Psychiatry 69: 514-519.

Gale EA, Gillespie KM (2001). Diabetes and gender. Diabetologia 44: 3-15.

Gianfrancesco FD, Grogg AL, Mahmoud RA, Wang RH, Nasrallah HA (2002). Differential effects of risperidone, olanzapine, clozapine, and conventional antipsychotics on type 2 diabetes: findings from a large health plan database. J Clin Psychiatry 63: 920-930.

Haffner SM, Lehto S, Ronnemaa T, Pyorala K, Laakso M (1998). Mortality from coronary heart disease in subjects with type 2 diabetes and in nondiabetic subjects with and without prior myocardial infarction. $N$ Engl J Med 339: 229-234.

Hagg S, Joelsson L, Mjorndal T, Spigset O, Oja G, Dahlqvist R (1998). Prevalence of diabetes and impaired glucose tolerance in patients treated with clozapine compared with patients treated with conventional depot neuroleptic medications. J Clin Psychiatry 59: 294-299.

Henderson DC, Copeland PM, Borba CP, Daley TB, Nguyen DD, Cagliero E et al (2006). Glucose metabolism in patients with schizophrenia treated with olanzapine or quetiapine: a frequently sampled intravenous glucose tolerance test and minimal model analysis. J Clin Psychiatry 67: 789-797.

Houseknecht KL, Robertson AS, Zavadoski W, Gibbs EM, Johnson DE, Rollema H (2007). Acute effects of atypical antipsychotics on whole-body insulin resistance in rats: implications for adverse metabolic effects. Neuropsychopharmacology 32: 289-297.

Kahn RS, Fleischhacker WW, Boter H, Davidson M, Vergouwe Y, Keet IP et al (2008). Effectiveness of antipsychotic drugs in firstepisode schizophrenia and schizophreniform disorder: an open randomised clinical trial. Lancet 371: 1085-1097.

Kane J, Honigfeld G, Singer J, Meltzer H (1988). Clozapine for the treatment-resistant schizophrenic. A double-blind comparison with chlorpromazine. Arch Gen Psychiatry 45: 789-796.

Kinon BJ, Stauffer VL, Kollack-Walker S, Chen L, Sniadecki J (2008). Olanzapine versus aripiprazole for the treatment of agitation in acutely ill patients with schizophrenia. J Clin Psychopharmacol 28: 601-607.

Koopman RJ, Mainous III AG, Diaz VA, Geesey ME (2005). Changes in age at diagnosis of type 2 diabetes mellitus in the United States, 1988-2000. Ann Fam Med 3: 60-63.

Laursen TM, Labouriau R, Licht RW, Bertelsen A, Munk-Olsen T, Mortensen PB (2005). Family history of psychiatric illness as 
a risk factor for schizoaffective disorder: a Danish register-based cohort study. Arch Gen Psychiatry 62: 841-848.

Lorenzo C, Okoloise M, Williams K, Stern MP, Haffner SM (2003). The metabolic syndrome as predictor of type 2 diabetes. Diabetes Care 26: 3153-3159.

Marder SR, Essock SM, Miller AL, Buchanan RW, Casey DE, Davis JM et al (2004). Physical health monitoring of patients with schizophrenia. Am J Psychiatry 161: 1334-1349.

Meyer JM, Davis VG, Goff DC, McEvoy JP, Nasrallah HA, Davis SM et al (2008). Change in metabolic syndrome parameters with antipsychotic treatment in the CATIE Schizophrenia Trial: prospective data from phase 1. Schizophr Res 101: 273-286.

Meyer JM, Stahl SM (2009). The metabolic syndrome and schizophrenia. Acta Psychiatr Scand 119: 4-14.

Moore TA, Buchanan RW, Buckley PF, Chiles JA, Conley RR, Crismon ML et al (2007). The Texas Medication Algorithm Project antipsychotic algorithm for schizophrenia: 2006 update. J Clin Psychiatry 68: 1751-1762.

Morrato EH, Druss B, Hartung DM, Valuck RJ, Allen R, Campagna $\mathrm{E}$ et al (2010). Metabolic testing rates in 3 state Medicaid programs after FDA warnings and ADA/APA recommendations for second-generation antipsychotic drugs. Arch Gen Psychiatry 67: 17-24.

Munk-Jorgensen P, Mortensen PB (1997). The danish psychiatric central register. Dan Med Bull 44: 82-84.

Narayan KMV, Boyle JP, Thompson TJ, Sorensen SW, Williamson DF (2003). Lifetime risk for diabetes mellitus in the United States. JAMA 290: 1884-1890.

Newcomer JW (2005). Second-generation (atypical) antipsychotics and metabolic effects: a comprehensive literature review. CNS Drugs 19(Suppl 1): 1-93.

Newcomer JW, Haupt DW, Fucetola R, Melson AK, Schweiger JA, Cooper BP et al (2002). Abnormalities in glucose regulation during antipsychotic treatment of schizophrenia. Arch Gen Psychiatry 59: 337-345.

Nielsen J, Dahm M, Lublin H, Taylor D (2009). Psychiatrists' attitude towards and knowledge of clozapine treatment. J Psychopharmacol published online on 22 January 2009 doi:10.1177/0269881108100320.
Pedersen CB, Mortensen PB (2001). Evidence of a dose-response relationship between urbanicity during upbringing and schizophrenia risk. Arch Gen Psychiatry 58: 1039-1046.

Qin P, Nordentoft M (2005). Suicide risk in relation to psychiatric hospitalization: evidence based on longitudinal registers. Arch Gen Psychiatry 62: 427-432.

Ramaswamy K, Masand PS, Nasrallah HA (2006). Do certain atypical antipsychotics increase the risk of diabetes? A critical review of 17 pharmacoepidemiologic studies. Ann Clin Psychiatry 18: 183-194.

Robinson DG, Woerner MG, Alvir JM, Geisler S, Koreen A, Sheitman B et al (1999). Predictors of treatment response from a first episode of schizophrenia or schizoaffective disorder. Am J Psychiatry 156: 544-549.

Sharpe JK, Stedman TJ, Byrne NM, Wishart C, Hills AP (2006). Energy expenditure and physical activity in clozapine use: implications for weight management. Aust $N Z J$ Psychiatry 40: 810-814.

Smith M, Hopkins D, Peveler RC, Holt RI, Woodward M, Ismail K (2008). First- v. second-generation antipsychotics and risk for diabetes in schizophrenia: systematic review and meta-analysis. Br J Psychiatry 192: 406-411.

Stahl SM, Mignon L, Meyer JM (2009). Which comes first: atypical antipsychotic treatment or cardiometabolic risk? Acta Psychiatr Scand 119: 171-179.

Taylor DM, Young C, Paton C (2003). Prior antipsychotic prescribing in patients currently receiving clozapine: a case note review. J Clin Psychiatry 64: 30-34.

Tiihonen J, Lonnqvist J, Wahlbeck K, Klaukka T, Niskanen L, Tanskanen A et al (2009). 11-year follow-up of mortality in patients with schizophrenia: a population-based cohort study (FIN11 study). Lancet 374: 620-627.

WHO (2009). World Health Organization, Collaborating Centre Drug Statistics Methodology http://www.whocc.no/atc_ddd_ index/.

Yood MU, DeLorenze G, Quesenberry Jr CP, Oliveria SA, Tsai AL, Willey VJ et al (2009). The incidence of diabetes in atypical antipsychotic users differs according to agent - results from a multisite epidemiologic study. Pharmacoepidemiol Drug Saf 18: 791-799. 